\title{
Transition region counterpart of a moving magnetic feature
}

\author{
C.-H. Lin ${ }^{1,2,3}$, D. Banerjee ${ }^{4}$, E. O'Shea ${ }^{1}$, and J. G. Doyle ${ }^{1}$ \\ 1 Armagh Observatory, College Hill, Armagh BT61 9DG, North Ireland, UK \\ e-mail: chl@arm.ac.uk \\ 2 National Center for Theoretical Sciences, Physics division, National Tsing-Hua University, Hsinchu, Taiwan \\ 3 Astronomy Department, Yale University, New Haven, CT06511, USA \\ 4 Indian Institute of Astrophysics, Koramangala, Bangalore 560034, India
}

Received 24 May 2006 / Accepted 30 August 2006

ABSTRACT

\begin{abstract}
Context. While moving magnetic features have been studied extensively at the photospheric level, the effect they have on the upper atmosphere remains largely unknown, and it is this which we seek to address in this work.

Aims. In this work we aim to investigate the chromospheric and transition-region dynamics associated with a moving magnetic monopole by using spectral time-series and images.

Methods. Cross correlation was applied to images taken by different instruments and at different times in order to spatially correlate brightenings seen at transition region temperatures with moving magnetic features seen in magnetograms. We used wavelet analysis to examine and compare the periodicities of time-series signals in different regions.

Results. Oscillations with a multitude of frequencies are found in the chromospheric and transition-region brightenings associated with a moving magnetic monopole. The region of the brightenings shows a tendency to be blue-shifted when compared to the average motion of the entire field of view. The results indicate the presence of waves and/or flows carrying energy from the monopole to the higher atmosphere.

Conclusions. We studied the influence of a moving magnetic monopole, as recorded by magnetograms, up to transition region temperatures. This suggests that the magnetic monopole, despite being small, can influence dynamics in the upper atmospheric layers.
\end{abstract}

Key words. Sun: granulation - Sun: magnetic fields - Sun: oscillations - Sun: chromosphere - Sun: transition region

\section{Introduction}

More than 35 years ago, Sheeley (1969) discovered, in spectroheliograms at $\lambda=3883 \AA$, a steady flow of bright points moving outward from a sunspot. These moving bright points were later seen in the magnetograms by Harvey \& Harvey (1973), who named the features as moving magnetic features (MMFs). The authors suggested that the features were magnetic knots resulting from the interactions between the sunspot magnetic field and the mass motion of supergranules. MMFs are very small $(<1500 \mathrm{~km})$, and can be either bipolar or unipolar. They often emerge near the outer edge of a penumbra, and move radially outward through an area with weak line-of-sight magnetic fields. However, magnetic features migrating inward to the umbra have also been observed (Ravindra et al. 2004). Zhang et al. (1992) reported that unipolar MMFs carry net flux from the sunspot while bipolar MMFs do not. Earlier observations by Lee (1992) found that the orientation of MMFs is random with no correlation with the polarity of the sunspot. The result was in agreement with the scenario of detached flux tubes, first suggested by Harvey \& Harvey (1973); i.e., a flux tube perturbed by the outward supergranulation flow is detached from the umbra, and floats to the surface while being pulled outward by the flow. The twists and the kinks of the tube then appear as the randomly oriented small magnetic features moving away from the umbra. However, more recent studies by Yurchyshyn et al. (2001) and Zhang et al. (2003) found that the leading pole in a bipolar MMF often has the same polarity as the sunspot. Based on their results, the authors suggested that MMFs are formed when the sunspot field lines in a small part of the canopy dip down to produce a U-loop. To date, all the studies of MMFs have focused on the properties of the feature. An extensive statistical study of all the properties of MMFs was recently carried out by Hagenaar \& Shine (2005) using the high-resolution Michelson Doppler Imager (MDI) magnetograms and the white-light images of MDI and TRACE (Transition Region and Coronal Explorer). In this paper, we will look at a different aspect of MMFs, that is, their effect on the higher atmosphere. We detected wave propagation and an indication of energy deposit in the transition zone, at the location above a unipolar MMF. Our analysis shows that the phenomena in the transition region are spatially and temporally correlated to the existence of the MMF, suggesting that the MMF, despite being small, is capable of influencing the dynamic behaviour in the atmosphere at least up to the transition region.

\section{Observation and data calibration}

We utilized time series from the Normal Incidence Spectrometer (NIS) of CDS/SoHO, images from MDI/SoHO, TRACE, EIT (Extreme ultraviolet Imaging Telescope), and raster images from NIS/CDS to study oscillatory behaviour in the transition region above a moving magnetic monopole. The time series correspond to the dataset s29536r00. It was a 95-min time-series observation taken on 2004 Feb. 13 at 07:59-09:35 UT. During the observation, the pointing of the detector was fixed in space while the Sun rotated under the field of view (FOV). The exposure time is $30 \mathrm{~s}$, and the cadence is approximately $37 \mathrm{~s}$. The details of the different image observations are listed in Table 1. 
Table 1. The details of the image observations.

\begin{tabular}{cccc}
\hline \hline Instrument/Observation & Wavelength (Spectral line) & Covered time period & Pixel size \\
\hline MDI & magnetogram & 2004-Feb.-13 06:00-08:30 UT & $2^{\prime \prime}$ \\
MDI & magnetogram & 2004-Feb.-13 13:53-13:59 UT & $2^{\prime \prime}$ \\
TRACE & $1600 \AA$ channel & 2004-Feb.-13 08:30 UT & $0.5^{\prime \prime}$ \\
TRACE & $1600 \AA$ channel & 2004-Feb.-13 13:40 UT & $0.5^{\prime \prime}$ \\
EIT & $304 \AA$ (He II) & 2004-Feb.-13 07:13 UT & $2.6^{\prime \prime}$ \\
NIS/CDS (s29535r00) & $629.7 \AA(\mathrm{O}$ v) & 2004-Feb.-13 07:37-07:59 UT & $4^{\prime \prime}$ \\
NIS/CDS (s29535r00) & $522.2 \AA$ (He I) & 2004-Feb.-13 07:37-07:59 UT & $4^{\prime \prime}$ \\
NIS/CDS (s29537r00) & $629.7 \AA(\mathrm{O} \mathrm{v})$ & 2004-Feb.-13 14:24-14:46 UT & $4^{\prime \prime}$ \\
NIS/CDS (s29537r00) & $522.2 \AA(\mathrm{He} \mathrm{I})$ & 2004-Feb.-13 14:24-14:46 UT & $4^{\prime \prime}$ \\
\hline
\end{tabular}

NIS/CDS data were calibrated by the most up-to-date standard calibration routines in SolarSoft ${ }^{1}$, with the offset between NIS1 and NIS2 corrected using the routine nis_rotate. As explained in CDS Software Note No. $53^{2}$, NIS/CDS line profiles were broadened after SoHO's recovery in October 1998. Therefore, each spectral line was fitted with a broadened Gaussian (BGauss) function, and the line intensity was computed accordingly. The NIS raster images and time series of individual lines were then constructed from the line intensity. The errors in the line intensities were computed based on the equations in CDS Software Note No. 49.

The noise and cosmic ray contamination in the TRACE images were reduced by a number of de-spike, de-streak, and smoothing routines. The orientations of the EIT and MDI images and the NIS raster images were corrected for the effect of the SoHO instruments being turned $180^{\circ}$ during our observation. After all the maps are calibrated, the coordinate difference between maps from the same instrument was corrected by the function coreg_map, and the coordinate difference between maps from different instruments was determined from a cross correlation between maps corresponding to a similar temperature. Due to the limited resolution of NIS/CDS maps, the uncertainty of the coordinate determination in this work is approximately $7^{\prime \prime}$. To be consistent, all the maps shown in this paper were shifted to 07:59 UT, which is the starting time of the NIS/CDS time-series observation s29536r00.

\section{Time-series analysis}

The oscillations were extracted from the time-series signals by subtracting the trend from the intensity variation. The process is called de-trending. The slit width of our NIS/CDS detector is $4 "$. Based on the solar rotation rate at the pointing of the detector, a feature would cross the slit in 25 min. Hence, an oscillation source of size $\approx 4^{\prime \prime}$ with periods longer than $12.5 \mathrm{~min}$ would leave our FOV before the second cycle is complete, and cannot be identified with confidence. Therefore, the trend of our time-series data is computed by a 25-pt running average to remove the periodicities of $15 \mathrm{~min}$ and longer. The amplitudes of an oscillation can be affected by the intensity of a spectral line. In other words, the amplitudes in a bright region can be greater than in a dark region simply because of the difference in the photon numbers the detector received in the two regions. To reduce the amplitude difference due to the line intensity and to prevent the oscillations in the dark region being obscured by the oscillations in the bright region, we divided the oscillations by the trend to produce what are called relative oscillations. In contrast, the

\footnotetext{
1 http://www.lmsal.com/solarsoft/

2 http://solar.bnsc.rl.ac.uk/
}

oscillations without being divided by the trend are called absolute oscillations.

The properties of the oscillations (e.g.periods, amplitudes, etc.) were determined by the use of wavelet transforms. A wavelet transform, being capable of revealing the temporal dependence of a signal, is more suitable than the traditional Fourier transform to analyze the oscillations that vary over time. The wavelet we used is the standard Morlet wavelet. To determine whether or not the oscillations are above the noise level, we implemented a randomization method (Linnell Nemec \& Nemec 1985) to estimate the significance level of the peaks in the wavelet spectrum. Details on the wavelet analysis may be found in Torrence \& Compo (1998) and O'Shea et al. (2001).

When a signal is composed of multiple oscillations with very different intensities, the weaker ones will be obscured by the stronger ones. To reveal these weaker components of the signal, we filtered out the stronger modes by removing the corresponding wavelet scales. In this paper, we applied two filters, P700 and P300, which filter out periods longer than $700 \mathrm{~s}$ and $300 \mathrm{~s}$, respectively. The filtered signals are called P700-filtered and P300-filtered. We also tried out other filters, but the resulting power was not significant enough. To infer the motion of material, we computed the relative Doppler velocity $\left(v_{\mathrm{d}_{i}}\right)$ at each pixel $i$ as follows:

$v_{\mathrm{d}_{i}}=c \times\left(\lambda_{i}-\lambda_{\mathrm{av}}\right) / \lambda_{\mathrm{av}}$,

where $c$ is the speed of light, $\lambda_{i}$ is the wavelength at pixel $i$, and $\lambda_{\mathrm{av}}$ is the average wavelength of the data set. Hence, $v_{\mathrm{d}_{i}}$ represents the motion of a point relative to the average motion of the area covered by the observation, and does not necessarily reflect the absolute velocity of the material at the location.

\section{Results}

\subsection{Signatures of the MMF at different temperatures}

The region of our observation is active region AR0554. A representative magnetogram of the region is shown in Fig. 1. The two white contours illustrate the umbra and penumbra. The sunspot can be seen surrounded by a ring devoid of strong magnetic fields along the line of sight, which can also be seen in Fig. 2. Within this ring, there are several small magnetic elements. From the series of magnetograms, we found that these elements are very dynamic. Here, we will examine one of the elements, which crossed the field of view (FOV) of our sit-and-stare observation.

This magnetic element has a positive magnetic polarity, presumably a monopole. It was moving away from the sunspot of negative polarity throughout the time period, 06:00-08:30 UT (cf. Fig. 2), and had disappeared in the second time series, 13:53-13:59 UT. To examine the influence of the magnetic 


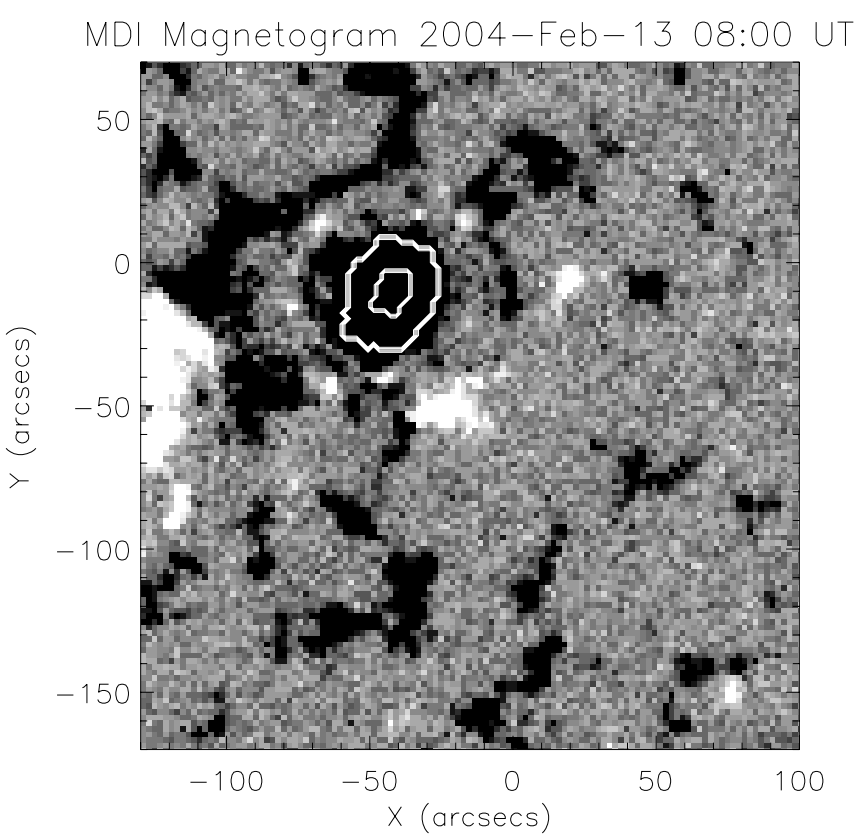

Fig. 1. A MDI magnetogram showing the magnetic configuration of active region AR0554. The sunspot is marked by the white contours.

monopole on the higher atmosphere, we show images formed at different temperatures in Fig. 3. Interestingly, a bright patch located at $\approx\left(-30^{\prime \prime}, 2^{\prime \prime}\right)$ was seen in the NIS/CDS He I $522.2 \AA$ and O V $629.7 \AA$ raster images at 07:37-07:59 UT and in the EIT He II $304 \AA$ image at 07:19 UT (the region in the white square in Fig. 3). Although the bright patch is not exactly at the coordinate of the monopole, the difference is within the resolution of NIS/CDS maps. In addition to the spatial correlation, the bright patch had disappeared in the later raster images at 14:24-14:46 UT, when the monopole also disappeared from the MDI magnetograms. Such coincidence in time indicates a possible connection between the existence of the moving magnetic element and the existence of the bright patch despite the absence of MDI data between 08:30 and 13:53 UT. In addition, we detected significant oscillations at the brightening location in the time series of He I 522.2 $\AA$ and O V $629.7 \AA$. The oscillations will be discussed in Sect. 4.2.1.

In Fig. 4, the contours of the magnetograms at different times are plotted over the TRACE $1600 \AA$ channel map taken at 08:30 UT. The two vertical dashed lines denote the beginning and end locations of the NIS time-series observations. The small circular contours traced by the two "L" shapes (from Solar $X \approx-33^{\prime \prime}$ to $-29^{\prime \prime}$ and Solar $Y \approx 2^{\prime \prime}$ to $7^{\prime \prime}$ ) correspond to the positions of the moving magnetic monopole at different times. Therefore, the monopole moved $\approx 4^{\prime \prime}$ in the $X$ direction and $\approx 5^{\prime \prime}$ in the $Y$ direction over the time period of $2.5 \mathrm{~h}$, which infers an apparent moving speed of $\approx 0.5 \mathrm{~km} \mathrm{~s}^{-1}\left(1^{\prime \prime}=715 \mathrm{~km}\right)$. The speed, direction of motion and the proximity to the sunspot allow us to identify this monopole as a moving magnetic feature.

The locations of this MMF at different times show that the MMF appears to move along the boundary of the supergranulation cell located at Solar $X \approx-50^{\prime \prime}$ to $-25^{\prime \prime}$, Solar $Y \approx 0^{\prime \prime}$ to $20^{\prime \prime}$. Comparing this TRACE $1600 \AA$ channel map with another map taken at a later time (cf. Fig. 5), we can see that this cell was broken by 13:40 UT, when the monopole also disappeared.
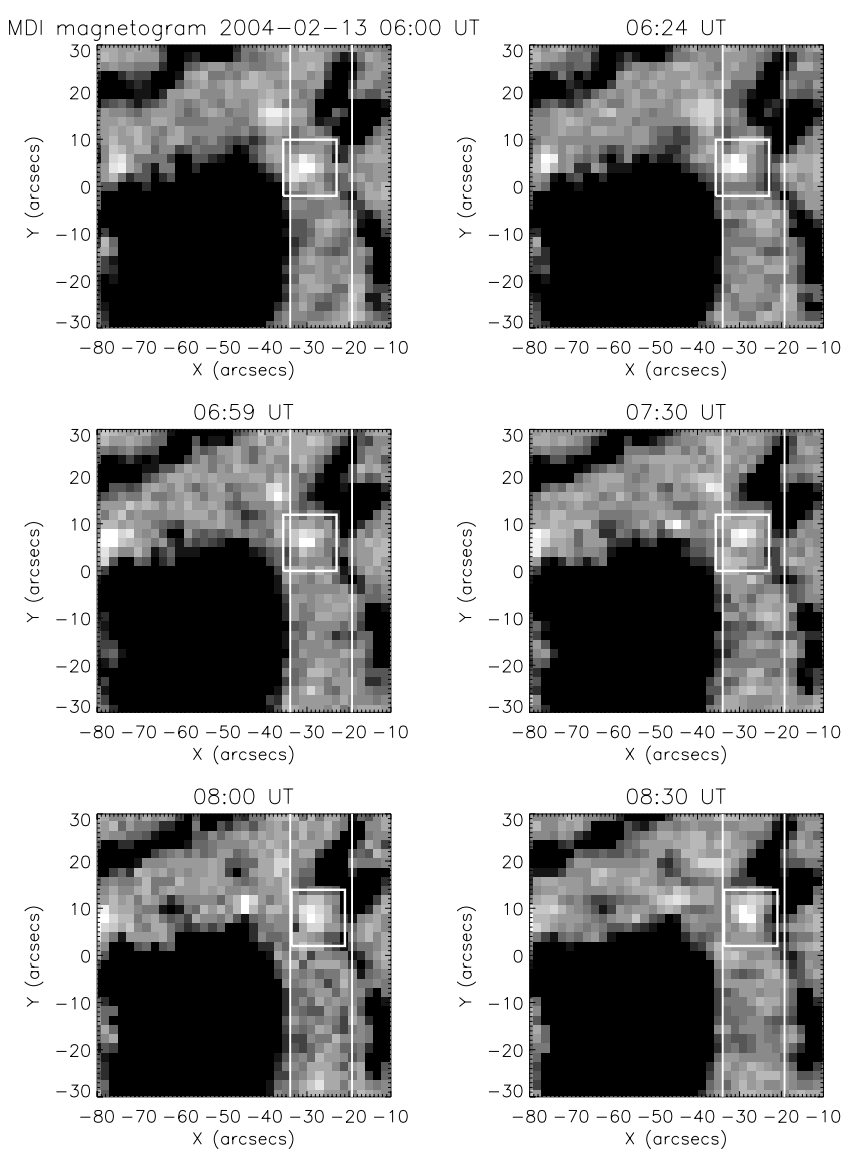

Fig. 2. MDI magnetograms taken at 06:00, 06:24, 06:59, 07:30, 08:00, and 08:30 UT, as indicated above each respective image. All the images have been shifted to the location corresponding to 07:59 UT, which is the observation starting time of the NIS/CDS time series s29536r00. In other words, the effect of solar rotation is removed. The area covered by this time-series observation is bounded between the two vertical lines. The white spot enclosed by the white square is the moving magnetic monopole discussed in this paper. The figure shows that this MMF was at approximately $\left(-29^{\prime \prime}, 4^{\prime \prime}\right)$ at 06:00 UT and moved to approximately $\left(-32^{\prime \prime}, 8^{\prime \prime}\right)$ at $08: 30 \mathrm{UT}$.

\subsection{The detected indication of waves}

To extract and examine the periodic features in the time series, we applied the de-trend procedure to remove the spatial effect. In addition, we calculated the relative Doppler velocity to examine the material motion in the MMF relative to the average motion of the entire FOV of the time series. The results are shown in Fig. 6. The location corresponding to the brightening seen in the NIS/CDS raster image and the MMF seen in MDI magnetograms (cf. Fig. 3) is enclosed in the white box, which covers Solar $Y=0^{\prime \prime}-13^{\prime \prime}$ and a 25-min observation duration from 08:35 UT to 09:00 UT. Based on the solar rotation rate at the location, a 25-min duration is equivalent to a distance of approximately 4 " in the Solar $X$ direction.

In the intensity time-series (the top panel), we can see a strong brightening in the area marked by the white box. The MMF contours in Fig. 3 show that this monopole is $\approx 4$ " across in the Solar X direction and that its location in Solar Y at 08:30 UT is $\approx 7^{\prime \prime}-10^{\prime \prime}$. Hence, this strong emission seen in the white box is likely correlated with the MMF within our spatial resolution. After the trend is subtracted from the time series, a periodic pattern can be seen in the white box (cf. second panel in Fig. 6). 

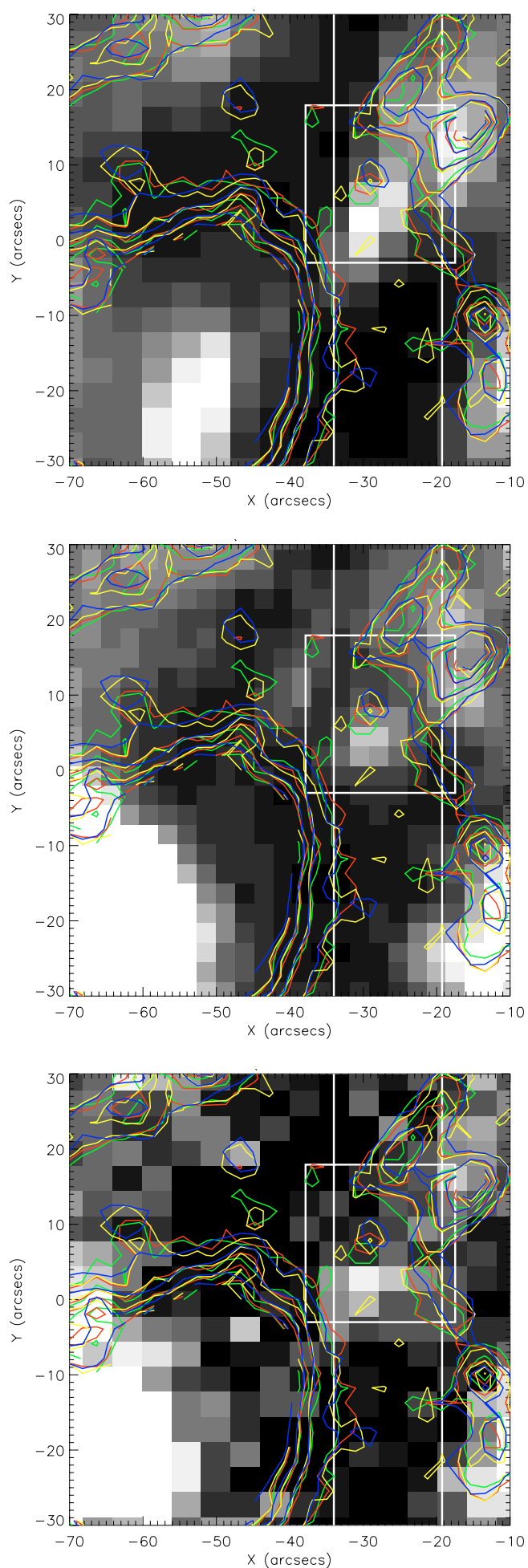

Fig. 3. The maps of NIS O v $629.7 \AA$ taken at 07:37 UT (top), EIT He II $304 \AA$ at 07:19 UT (middle), and NIS He I 522.2 $\AA$ at 07:37 UT (bottom). The MDI magnetograms taken at 06:59 UT (green), 07:30 UT (red), 08:00 UT (yellow) and 08:30 UT (blue) are over-plotted on all three maps to illustrate the variation of the magnetic fields, and, specifically, the motion of the MMF. All the maps were shifted to the location corresponding to 07:59 UT, which is the observation starting time of the NIS/CDS time series s29536r00. The area covered by this time-series observation is bounded between the two vertical lines. The white square encloses the region of the moving magnetic feature, indicated by three partially overlapping circular contours. All three maps, O v $629.7 \AA$, He II $304 \AA$, and He I $522.2 \AA$, show a bright patch touching the south end of the MMF.

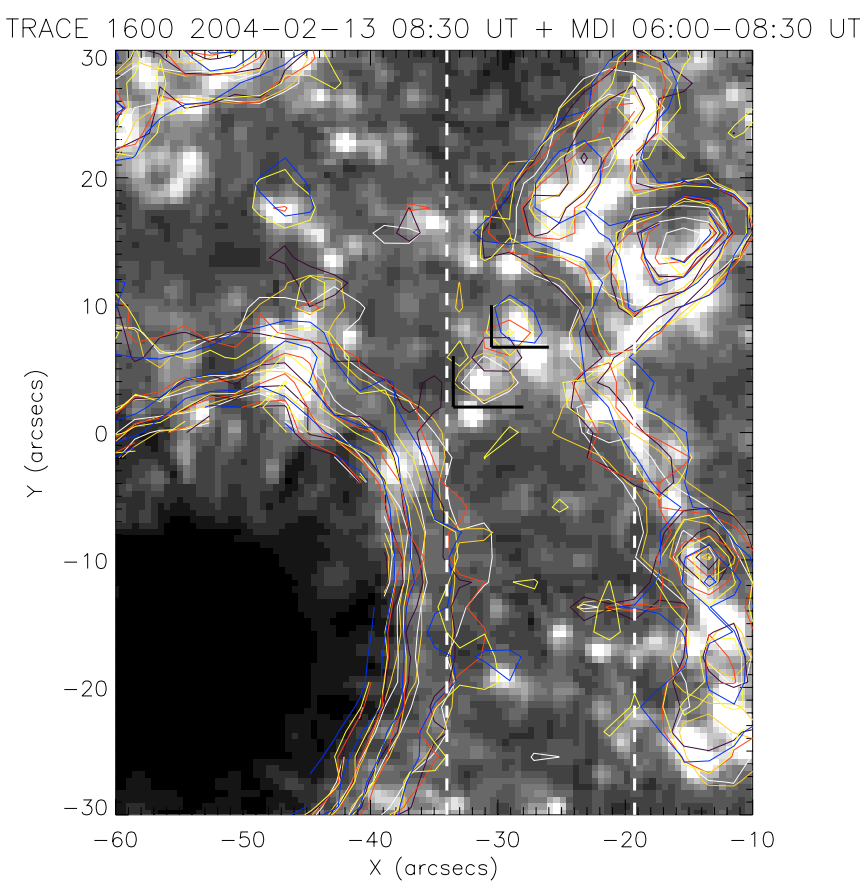

Fig. 4. The TRACE $1600 \AA$ channel image taken at 08:30 UT. The overplotted contours are the MDI magnetograms taken at 06:00 UT (white), 06:24 UT (orange), 06:59 UT (black), 07:30 UT (red), 08:00 UT (yellow) and 08:30 UT (blue). All the images have been shifted to the location corresponding to 07:59 UT, which is the observation starting time of the time series, NIS s29536r00. The area covered by this timeseries observation is bounded between the two vertical dashed lines. The thread of circular contours spreading from Solar $X \approx-33^{\prime \prime}$ to $-29^{\prime \prime}$ and Solar $Y \approx 2$ " to 11 ", as traced by the two "L" shapes, represents the locations of the moving magnetic monopole from 06:00-08:30 UT. The thread coincides with the boundary of a super-granulation cell (see also the white-square enclosed region in Fig. 5).

However, this pattern, consisting of only two cycles, may be a manifestation of spatial effects rather than a periodic signal. The examination of the possible temporal periodicity is discussed in Sect. 4.2.1. From the relative Doppler velocity (the bottom panel), a clear stripe of blue-shifts between $10^{\prime \prime}$ and $-5^{\prime \prime}$ can be seen during almost the entire observation period. Specifically, in the region of the white box, the blue shifts are localized between $7^{\prime \prime}$ and $0^{\prime \prime}$. We further examined the relative velocity at individual pixels. The results of the examination are discussed in Sect. 4.2.2.

\subsubsection{Oscillations}

We applied the wavelet analysis to examine the behaviour of the NIS/CDS $\mathrm{O} \mathrm{V}$ and He I time series over the moving magnetic monopole. From Fig. 3, we see that the region of interest extends from Solar $Y \approx-4^{\prime \prime}$ to $10^{\prime \prime}$, i.e.the upper and lower edge of the white square. In Fig. 7, we use the results of the pixel at Solar $Y \approx 3^{\prime \prime}$ (corresponding to pixel 20 of our CDS slit) to illustrate the common properties seen in the oscillations of the bright patch of $\mathrm{O} \mathrm{V}$. We would like to point out that the adjacent pixels also show similar oscillation pattern, whereas pixels 18 , 19,21 , and $22\left(\approx-5^{\prime \prime},-1^{\prime \prime}, 7^{\prime \prime}\right.$ and $\left.11^{\prime \prime}\right)$ show similar oscillations with $50 \%$ reduction in amplitude. The unfiltered intensity variation of pixel 20 along with the error bars is plotted in the top panel. The large intensity enhancement seen in the unfiltered signal between 30 and $55 \mathrm{~min}$ is a manifestation of the $\mathrm{O} \mathrm{V}$ 

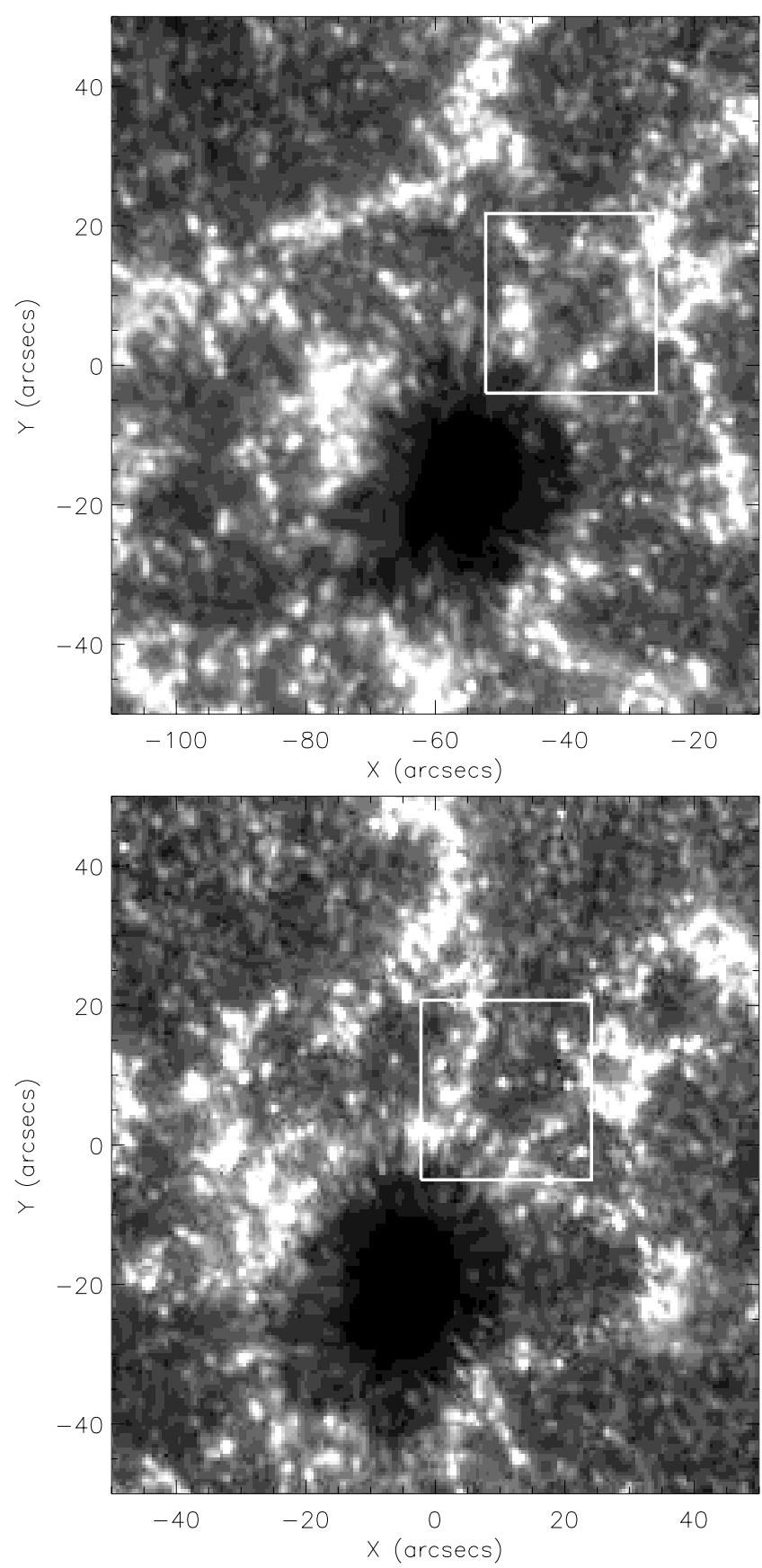

Fig. 5. TRACE $1600 \AA ̊$ channel image taken at 08:30 UT (upper panel) and 13:40 UT (lower panel).

brightening above the monopole (as seen in the raster image in Fig. 3) crossing the NIS/CDS detector. In other words, the rotation of the Sun brings the monopole to cross the NIS/CDS slit during our observation. The dip in the middle of the enhancement may be due to non-uniform brightness in the brightening. To extract the oscillations that reside on top of the intensity enhancement, we removed this dominant feature of the enhancement by applying the P700 filter (cf. Sect. 3 for details). We also applied the P300 filter in order to examine the oscillations with periods shorter than $300 \mathrm{~s}$. The wavelet analysis results of the two filtered relative oscillations are presented in the two sets of plots following the unfiltered signal in Fig. 7. In each set, the upper left panel shows the filtered relative oscillations, the wavelet spectrum is illustrated in the bottom panel, and the
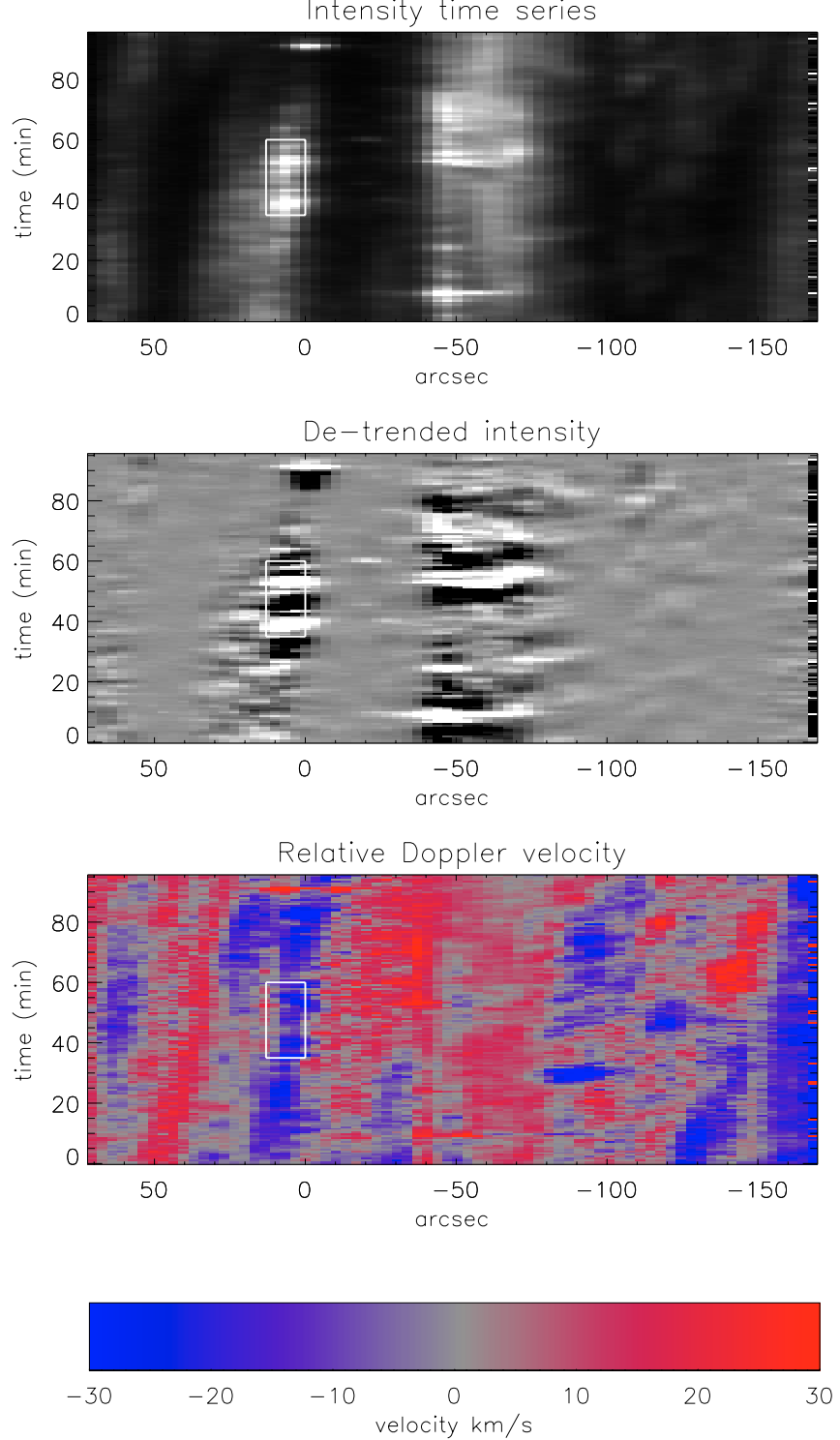

Fig. 6. The O v $629.7 \AA$ time series of the intensity variation (top), the de-trended intensity variation (middle), and the relative Doppler velocity with colour bar (bottom). The white rectangular marks the location of the MMF. The $X$ axis corresponds to the Solar $Y$ coordinates of the pixels along the detector slit, and the $Y$ axis shows the time.

global wavelet (i.e. the sum of the wavelet power over time at each oscillation period) is plotted in the lower right panel. The wavelet spectra are plotted in reversed colours; hence, the darker colours correspond to the higher powers. The white contours enclose periods of the oscillations that are above the $95 \%$ significance level as determined by the randomization method, and the hashed lines mark the region where the edge effect becomes significant (see Torrence \& Compo 1998). The relative oscillation plots show that the amplitudes inside the bright patch (30$65 \mathrm{~min}$ ) are almost twice as large as those outside (0-30 min). Hence, to obtain the oscillation properties outside the bright patch, we also examined these weaker oscillations separately in addition to the analysis of the entire signal.

The wavelet analysis results of P700-filtered and P300-filtered signals reveal that 5-min oscillations (i.e. periods between $250 \mathrm{~s}$ and $400 \mathrm{~s}$ ) are the dominant mode through the entire signal, both inside and outside of the MMF, while 


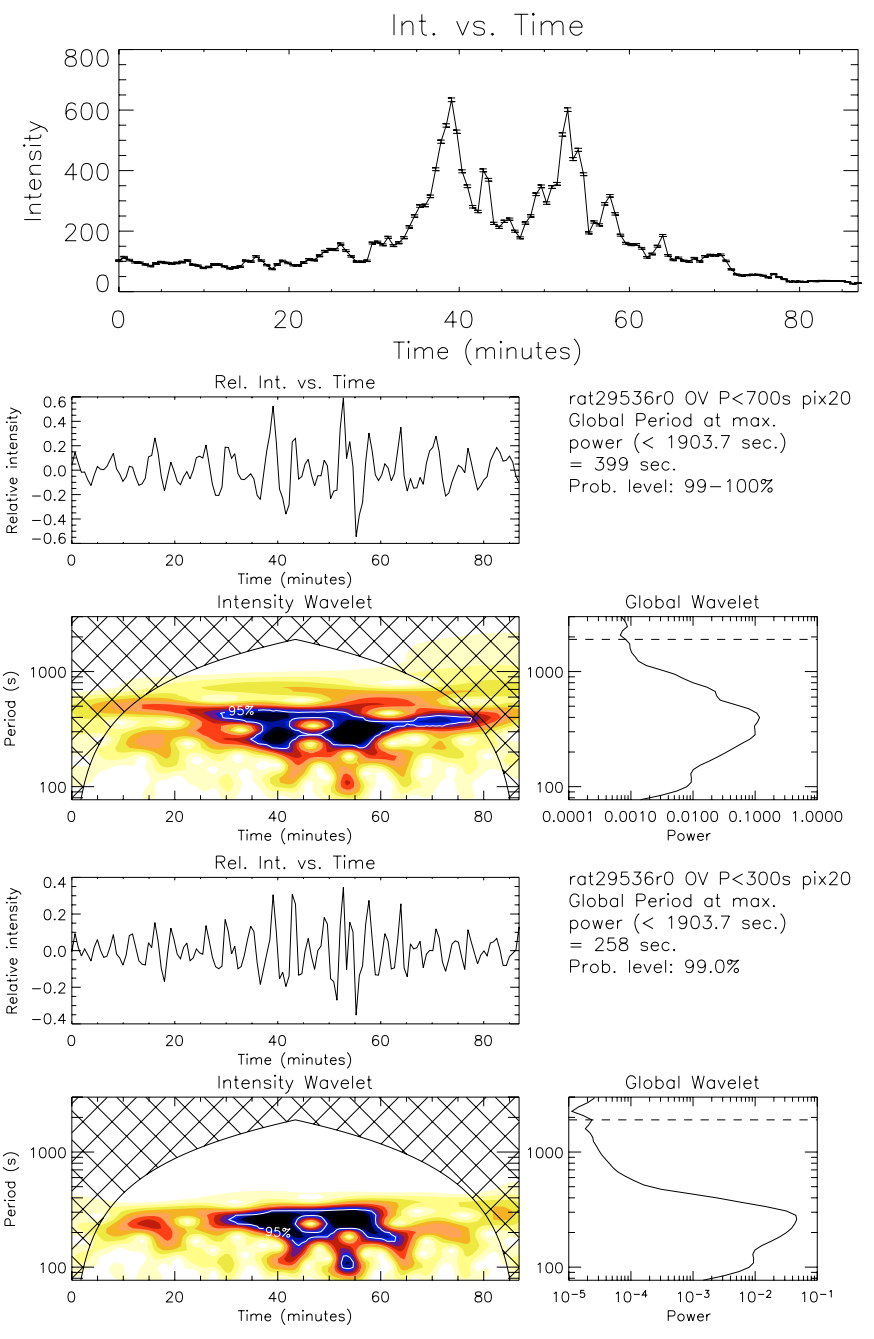

Fig. 7. The top row represents the intensity variation of O v $629.7 \AA$ at Solar $Y \approx 3^{\prime \prime}$. The errors are shown as vertical bars. The panels that follow are two sets of wavelet analysis of filtered relative intensity oscillations, P700-filtered (i.e., oscillations with periods longer than $700 \mathrm{~s}$ are filtered out) and P300-filtered, as marked.

a multitude of shorter-period oscillations only exist inside the MMF. Figure 7 demonstrates such an example: the P700-filtered wavelet spectrum shows that there are only two major period bands, 200-300 s and 400-500 s, for the oscillations before $30 \mathrm{~min}$ while the spectrum of the oscillations between 30 and $60 \mathrm{~min}$ extends from $100 \mathrm{~s}$ to $700 \mathrm{~s}$. When the modes of periods longer than $300 \mathrm{~s}$ are filtered out, the strongest oscillation in the $\mathrm{P} 300$-filtered signal often shows a periodicity $\approx 3 \mathrm{~min}$, and the oscillation is most significant for the time interval 30-65 min of the observing sequence, which corresponds to the transit time interval for the MMF across our CDS pixel. This result echoes the conclusion by Lin et al. (2005) that 3-min oscillations can exist outside of a sunspot in regions with strong magnetic fields.

Although the signals of He I $522.2 \AA$ are very weak, the wavelet analysis results of $\mathrm{He} \mathrm{I}$ are consistent with the results of O v $629.7 \AA$, that is, that a dominant 5-min oscillation is seen in the entire signal, but that a more complex spectrum is seen only inside the moving magnetic monopole. We should point out here that, in addition to a chromospheric contribution, He I $522.2 \AA$ can result from particles and radiation from the transition region and corona (Andretta \& Jones 1997). Hence, the features seen in
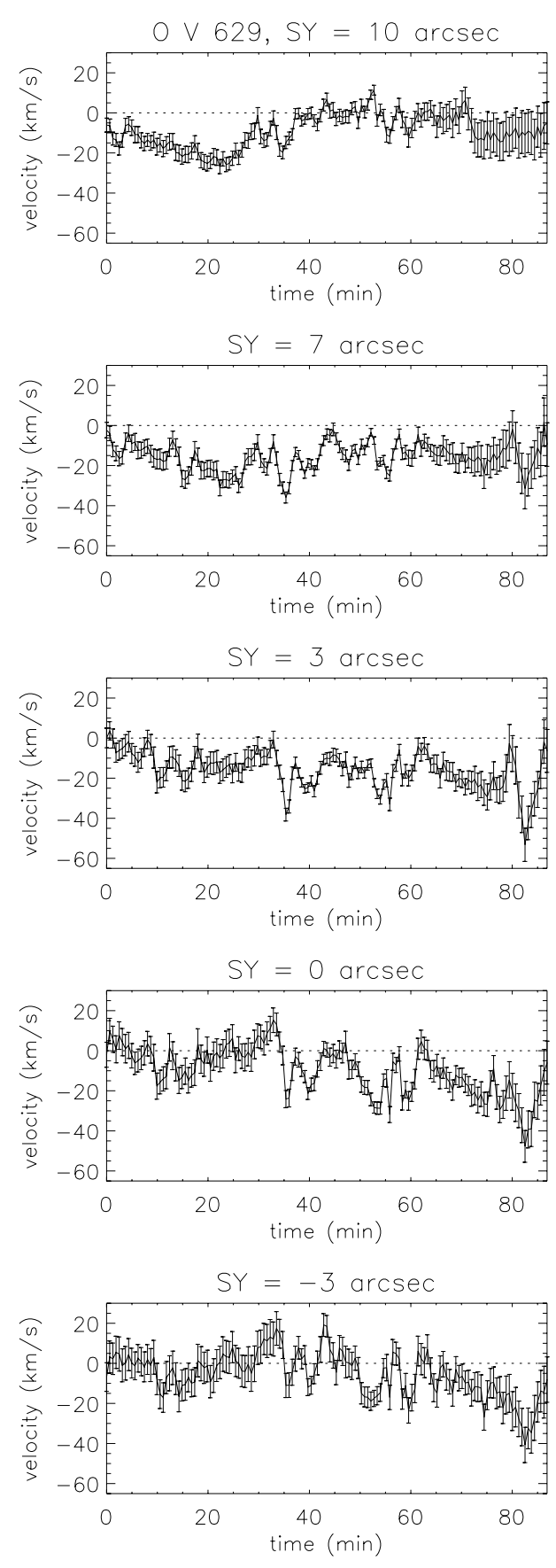

Fig. 8. The Doppler velocities of the NIS/CDS O v $629.7 \AA$ time series at selected pixels along the slit. The pixels are selected to cover the region over the magnetic monopole. The Solar $Y$ coordinate of each pixel is indicated above the corresponding plot. The blue shifts are represented by negative velocities. The plots show that there are blue shifts between 7 " and $0^{\prime \prime}$ during the time when the slit is over the magnetic monopole. $(\approx 35 \mathrm{~min}$ to $\approx 60 \mathrm{~min}$ as inferred from the unfiltered oscillations in Fig. 7).

He I $522.2 \AA$ reflect the collective conditions from both chromosphere and higher-temperature layers (O'Shea et al. 2002).

\subsubsection{Relative material motion}

In Fig. 8, we plot the relative Doppler velocities of O v $629.7 \AA$ vs. time at several pixels along the slit. The Solar $Y$ coordinates of the pixels are indicated above the corresponding plots. 
The dotted horizontal line marks $0 \mathrm{~km} \mathrm{~s}^{-1}$, and the negative velocities correspond to blue shifts. The bright patch over the magnetic monopole is between $35 \mathrm{~min}$ and $60 \mathrm{~min}$. We see that this $35-60 \mathrm{~min}$ region is relatively blue-shifted between $7^{\prime \prime}$ and $0^{\prime \prime}$ and becomes red-shifted below $0^{\prime \prime}$, which is also illustrated in Fig. 6. Intriguingly, while the intensity enhancement is localized in the region of MMF detection, large blue shifts are noticeable before and after the transition of the MMF, that is, between $7^{\prime \prime}$ and $10^{\prime \prime}$ during the first $20 \mathrm{~min}$ and between $3^{\prime \prime}$ and $-7^{\prime \prime}$ after 60 min of the sequence (see also Fig. 6). The relative Doppler velocities of He I 522.2 $\AA$ show the same pattern. Because the velocity is computed relative to the average velocity of the entire FOV, such blue shifts are not necessarily associated with an upward motion. Nevertheless, the shifts do indicate the existence of material flow. The long stripe of the blue shifts extending before and after the transition of the MMF may indicate an additional east-west structure guiding/confining the material motion.

\section{Discussions and conclusion}

During our observation on Feb. 13th, 2004, we detected a positive magnetic monopole (MMF) moving away from the sunspot. The path of the MMF followed the boundary of a supergranulation cell. The supergranular cell broke apart when the MMF was gone. The MMF also overlapped with a bright patch seen in both the chromosphere and transition region. The correlation between the MMF and the atmospheric brightening is further confirmed by the sudden and localized intensity enhancement seen in the $\mathrm{O} V$ intensity time-series during the transition of the monopole (cf. Fig. 6). The slight offset between this MMF and the bright patch seen in Fig. 3 is likely due to the error in our coordinate determination. However, it is also possible that the magnetic tubes become curved as they reach to the higher atmosphere and therefore the higher-temperature apex may appear spatially displaced relative to the lower-temperature footpoints, where the MMF is detected in the magnetograms. Such coincidence in space and time indicates a possible link between this moving monopole, the super-granulation boundary, and the strong emission in the chromosphere and transition region layers. While several papers (e.g. Harvey \& Harvey 1973; Meyer et al. 1974; Hagenaar \& Shine 2005) have discussed the effect of the super-granular flow on the formation and the travelling paths of MMFs, rather little has been done on the association between MMFs and the emissions above the photosphere.

Harvey \& Harvey (1973) noted very weak to absent H $\alpha$ emission from MMFs, while Penn \& Kuhn (1995) failed to detect MMFs in He I 10830. Hagenaar \& Shine (2003) using TRACE 171 data showed that coronal emission does not show an immediate response to the birth and disappearance of individual MMF and, therefore, that the role of MMF in the dynamics of upper layers of the atmosphere is unclear. Ryutova \& Hagenaar (2005) suggested that there are four types of MMFs: type I are compact pairs of opposite polarity elements that emerge anywhere in penumbra or moat region, moving radially outward with a velocity exceeding the velocity of ambient flows; type II are unipolar features of the same polarity as the sunspot, moving outward from the sunspot with higher velocities than type I; type III are unipolar features but have the polarity opposite to the sunspot's and travel with higher velocities than the other two types of MMFs; type IV appear as compact bipoles flowing into sunspots with an inner foot of a polarity opposite to the sunspot's. These different types may have quite different formation processes, e.g., the lack of a chromospheric component in some MMF's may be due to a U-loop submergence in part of the magnetic canopy. The MMF discussed here appears to be type III and may have resulted from a $\Omega$-loop emergence, expanding into the upper atmosphere as a result of shocks. Penn \& Kuhn (1995) have seen the bipolar nature of the photospheric magnetic field, whereas the chromospheric field appeared to be unipolar. They have also pointed out that their observed MMFs are linked with low lying loops which do not reach to the upper chromosphere where the He I is formed. These loops must be less than $1500 \mathrm{~km}$ high, which is the height where significant He I line formation begins, which explain why they could not identify the MMFs in the He I line. In recent work, Hagenaar \& Frank (2006) identified MMFs in TRACE $1600 \AA$ and studied their presence in high resolution filtergrams of $\mathrm{Ca}$ II K, G-band, $\mathrm{H} \alpha$, together with vector magnetograms. Their preliminary results suggest strong correlation between Ca II K and TRACE $1600 \AA$ with unsigned magnetic flux density. Clearly, more simultaneous observations of the photosphere and upper atmosphere of MMFs is required from further SoHO and/or Solar-B observations.

The wavelet analysis on the time series of $\mathrm{O} \mathrm{V}$ and $\mathrm{He} \mathrm{I}$ above the monopole reveals that there are significant oscillations in both lines in this region. The two lines show a similar oscillation pattern, indicating that the oscillations of the two lines are related or result from the same source. Although the temperature layer that He I represents is uncertain due to the complex formation mechanisms of the line, the detection confirms that the oscillations caused by MMF can be seen in the higher atmosphere.

The wavelet spectra in Fig. 7 show that a dominant 5-min oscillation runs through the whole time series, both inside and outside of the monopole region. However, the signal inside the monopole is composed of a wider range of oscillation modes than the signal outside, which is manifested by the multitude of strong wavelet peaks within the monopole region (from $\approx 35 \mathrm{~min}$ to $\approx 60 \mathrm{~min}$ ) and the relatively clean spectrum everywhere else. The dominant 5-min oscillations may be due to the global p-mode oscillations. Theoretical models to-date generally depict MMFs as magnetic knots or kinks resulting from sunspot magnetic tubes being strongly disturbed by the mass flows and buoyancy. These perturbations can excite a broad spectrum of modes, which can travel along the field lines to the higher atmosphere. There has not been any study regarding the temperature of the MMF magnetic structure. Our detection of transition-region oscillations spatially and temporally correlated with the existence of the MMF implies that MMF field lines may be at the temperature of the transition region or that the effects of the fields can reach up to the transition region.

From the relative Doppler velocities of the time series, we see that a east-west directed stripe over the location of the MMF is blue-shifted while the regions north of and immediately south of the MMF are relatively red shifted (cf. Fig. 6). Because the relative Doppler velocity only reflects the relative, not exact, motion of the material, the blue shifts could result from either a greater upward motion or a smaller downward motion. Nevertheless, Fig. 6 indicates that there are multiple regions with different material motions. Unlike the intensity enhancement, the relative blue shifts extend beyond the transition of the MMF, which could indicate an additional east-west directed structure effecting the material motion.

The aforementioned phenomena suggest that the MMFs, despite being small in size, could influence the dynamics in the atmosphere and may contribute to the heating of the upper layers, at least up to the transition region. Hence, it is necessary to conduct an extensive statistical search for the upper-atmospheric 
counterparts of MMF. Such a study could provide insights into the link between small photospheric magnetic dynamics and upper-atmospheric dynamics.

Acknowledgements. We would like to thank the CDS team for their help in obtaining and reducing the data and in particular B. Thompson for advice on the line blending issues. CDS, EIT, and MDI are part of SOHO, the Solar and Heliospheric Observatory, which is a project of international cooperation between ESA and NASA. This work was supported in part by a PRTLI research grant for Grid-enabled Computational Physics of Natural Phenomena (Cosmogrid). CHL was also supported by the grant NSC 94-2119-M-007-001 from the National Science Council in Taiwan. EOS is supported by PPARC grant number PP/D001129/1. We wish to thank the Royal Society and the British Council for funding visits between Armagh Observatory and the Indian Institute of Astrophysics. Research at Armagh Observatory is grant-aided by the N. Ireland Dept. of Culture, Arts and Leisure.

\section{References}

Andretta, V., \& Jones, H. P. 1997, ApJ, 489, 375

Hagenaar, H. J., \& Frank, Z. A. 2006, ESA SP-617: SOHO-17, in press
Hagenaar, H. J., \& Shine, R. A. 2003, AGU Fall Meeting Abstracts, B544

Hagenaar, H. J., \& Shine, R. A. 2005, ApJ, 635, 659

Harvey, K., \& Harvey, J. 1973, Sol. Phys., 28, 61

Lee, J. W. 1992, Sol. Phys., 139, 267

Lin, C.-H., Banerjee, D., Doyle, J. G., \& O'Shea, E. 2005, A\&A, 444, 585

Linnell Nemec, A. F., \& Nemec, J. M. 1985, AJ, 90, 2317

Meyer, F., Schmidt, H. U., Wilson, P. R., \& Weiss, N. O. 1974, MNRAS, 169, 35

O’Shea, E., Banerjee, D., Doyle, J. G., Fleck, B., \& Murtagh, F. 2001, A\&A, 368,1095

O'Shea, E., Muglach, K., \& Fleck, B. 2002, A\&A, 387, 642

Penn, M. J., \& Kuhn, J. R. 1995, ApJ, 441, L51

Ravindra, B., Venkatakrishnan, P., \& Kumar, B. 2004, Sol. Phys., 225, 47

Ryutova, M. P. \& Hagenaar, H. J. 2005, AGU Spring Meeting Abstracts, A4

Sheeley, N. R. 1969, Sol. Phys., 9, 347

Torrence, C., \& Compo, G. P. 1998, Bull. Am. Meteorolog. Soc., 79, 61

Yurchyshyn, V. B., Wang, H., \& Goode, P. R. 2001, ApJ, 550, 470

Zhang, H., Ai, G., Wang, H., Zirin, H., \& Patterson, A. 1992, Sol. Phys., 140, 307

Zhang, J., Solanki, S. K., \& Wang, J. 2003, A\&A, 399, 755 